\title{
A Comparison on the Bounds of Chromatic Preserving Number and Dom-Chromatic Number of Cartesian Product and Kronecker Product of Paths
}

T N Janakiraman* and M Poobalaranjani ${ }^{*}$

\section{Abstract}

Let $\mathrm{G}$ be a simple graph with vertex set $\mathrm{V}$ and edge set $\mathrm{E}$. A Set $\mathrm{S} \subseteq \mathrm{V}$ is said to be a chromatic preserving set or a cp-set if $\chi(<S>)=\chi(G)$ and the minimum cardinality of a $c p$-set in $G$ is called the chromatic preserving number or cp-number of $G$ and is denoted by cpn(G). A cp-set of cardinality $\operatorname{cpn}(\mathrm{G})$ is called a cpn-set. A subset $\mathrm{S}$ of $\mathrm{V}$ is said to be a dom- chromatic set (or a dc-set) if $S$ is a dominating set and $\chi(<S>)=\chi(G)$. The minimum cardinality of a dom-chromatic set in a graph $G$ is called the dom-chromatic number (or dc- number) of $G$ and is denoted by $\gamma_{c h}(G)$. The Kronecker product $G_{1} \wedge G_{2}$ of two graphs $G_{1}=\left(V_{1}, E_{1}\right)$ and $G_{2}=\left(V_{2}, E_{2}\right)$ is the graph $G$ with vertex set $V_{1} \times V_{2}$ and any two distinct vertices $\left(u_{1}, v_{1}\right)$ and $\left(u_{2}, v_{2}\right)$ of $G$ are adjacent if $u_{1} u_{2} \in E_{1}$ and $v_{1} v_{2} \in E_{2}$. The Cartesian product $G_{1} \times G_{2}$ is the graph with vertex set $\mathrm{V}_{1} \times \mathrm{V}_{2}$ where any two distinct vertices $\left(\mathrm{u}_{1}, \mathrm{v}_{1}\right)$ and $\left(\mathrm{u}_{2}, \mathrm{v}_{2}\right)$ are adjacent whenever (i) $u_{1}=u_{2}$ and $v_{1} v_{2} \in E_{2}$ or (ii) $u_{1} u_{2}$ $\in \mathrm{E}_{1}$ and $\mathrm{v}_{1}=\mathrm{v}_{2}$. These two products have no common edges. They are almost like complements but not exactly. In this paper, we discuss the behavior of the cp-number and dc-number and their bounds for product of paths in the two cases. A detailed comparative study is also done.

* Department of Mathematics, National Institute of Technology, Tiruchirapalli-620015, India, tnjraman2000@yahoo.com; janaki@nitt.edu

† Seethalakshmi Ramaswami College, Tiruchirapalli-620002, India; mpranjani@hotmail.com

Received: July 2012, Reviewed: Aug. 2012 
Keywords: Cp-set, cp-number, dom-chromatic set, dom-chromatic number, Kronecker product, Cartesian product.

\section{Introduction}

A dominating set $\mathrm{S}$ is a subset of vertices in a graph $\mathrm{G}=(\mathrm{V}, \mathrm{E})$ such that every vertex in $G$ either belongs to $S$ or has a neighbor in $S$. The minimum cardinality of a dominating set of $G$ is called the domination number of $\mathrm{G}$, denoted by $\mathrm{\gamma}(\mathrm{G})$. A proper coloring of a graph $G$ is a function from the vertices of $G$ to a set of colors such that any two adjacent vertices have distinct colors. The chromatic number of a graph $G$ is the minimum number of colors needed for a proper coloring of $G$ and is denoted by $\chi(G)$. If $\chi(G)=k$, then $G$ is said to be $k$-chromatic. Both coloring and domination problems have many applications in the real life situations. Though the two problems are studied independently and extensively, not much research has been done on combining domination and coloring. We made an attempt in combining these two parameters. A dominating set $\mathrm{S}$ is said to be a conditional dominating set if the subgraph induced by $\mathrm{S}$ satisfies a given condition. Combining the concepts of domination and coloring, we define a new conditional dominating set in [1] and [2], termed as dom-chromatic set. A set $S$ $\subseteq \mathrm{V}(\mathrm{G})$ is said to be a dom-chromatic set (or a dc-set) of $\mathrm{G}$, if $\mathrm{S}$ is a dominating set of $G$ and $\chi(<S>)=\chi(G)$. The minimum cardinality of a dom-chromatic set of $G$ is called the dom-chromatic number (or dc-number) of $\mathrm{G}$ and is denoted by $\gamma_{\mathrm{ch}}(\mathrm{G})$.

In general, a dc-set can be extracted in two ways: (1) Find a minimum dominating set S. If $\chi(<S>) \neq \chi(G)$, add minimum number of vertices to $S$ until the chromatic number of the subgraph induced by these vertices is same as the chromatic number of the graph (2) Find a smallest subset $S$ of $V$ such that $\chi(<S>)=\chi(G)$. Add minimum number of vertices to $S$ until $S$ dominates $G$. Though a dc-set can be obtained for a given graph in these two ways, second method is more effective as it involves the concept of critical graphs. The concept of critical graphs was introduced by Dirac in [3]. He defined a graph $G$ to be a critical graph if $\chi(<G>)<\chi(G-v)$ for each vertex $v$ of $G$. The complete graph $K_{2}$ is an example of a 
2-critical graph and odd cycles are 3-critical graphs. Thus, to find a minimum dc-set of a given graph $\mathrm{G}$, first it is necessary to find a smallest critical subgraph whose chromatic number is the same as that of G. This motivated us to define a new set called a chromatic preserving set or a cp-set [4]. A Set $S$ of vertices is said to be a chromatic preserving set or a $c p$-set if $\chi(<S>)=\chi(G)$ and the minimum cardinality of a cp-set in $\mathrm{G}$ is called the chromatic preserving number or cp-number of $\mathrm{G}$ and is denoted by $\operatorname{cpn}(\mathrm{G})$.

For the graph in figure 1, the shaded vertices form both a dc-set and a cp-set. It can be verified that the dc-number and cp-number of the graph is 3 .

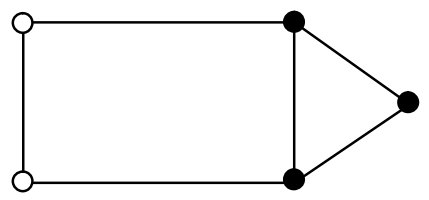

Figure 1

In this paper, we compare the cp-number, dc-number and their bounds for Cartesian product and Kronecker product of paths. Generally, graph products lead to varieties of new graphs. Imrich and Izbicki [5] have shown that there exist exactly twenty products of graphs defined on the Cartesian product of the vertex sets of the two factor graphs, where the adjacencies of the vertices in the product, depend only on the adjacencies in the two factor graphs. Weichsel [6] defined the Kronecker product $\mathrm{G}_{1} \wedge \mathrm{G}_{2}$ of two graphs $G_{1}=\left(V_{1}, E_{1}\right)$ and $G_{2}=\left(V_{2}, E_{2}\right)$ as the graph with vertex set $V_{1} \times V_{2}$ in which any two distinct vertices $\left(\mathrm{u}_{1}, \mathrm{v}_{1}\right)$ and $\left(\mathrm{u}_{2}, \mathrm{v}_{2}\right)$ of $\mathrm{G}$ are adjacent if $\mathrm{u}_{1} \mathrm{u}_{2} \in \mathrm{E}_{1}$ and $\mathrm{v}_{1} \mathrm{v}_{2} \in \mathrm{E}_{2}$. It has been shown that the Kronecker product is a good choice to construct lager networks and has received much research attention recently. The Cartesian product $\mathrm{G}_{1} \times \mathrm{G}_{2}$ of two graphs $\mathrm{G}_{1}$ and $\mathrm{G}_{2}$ was first defined by Shapiro [7] as the graph with vertex set $\mathrm{V}_{1} \times \mathrm{V}_{2}$ and any two distinct vertices $\left(u_{1}, v_{1}\right)$ and $\left(u_{2}, v_{2}\right)$ are adjacent only if either $u_{1}=u_{2}$ and $v_{1} v_{2} \in E_{2}$ or $\mathrm{u}_{1} \mathrm{u}_{2} \in \mathrm{E}_{1}$ and $\mathrm{v}_{1}=\mathrm{v}_{2}$. Sabidussi [8] and Teh and Yap [9] also independently studied this product. Domination number of the Cartesian product of two graphs has received much attention, with a main objective to prove the well-known Vizing's conjecture. The 
conjecture states that the domination number of the Cartesian product of two graphs is at least as large as the product of the respective domination numbers. It remains an open problem. The conjectured bound has been verified for many large classes of graphs. Vizing's conjecture has inspired many researchers to investigate the possibility of similar bounds for other domination parameters as well as other graph products.

From the definitions of the products, it is clear that any edge of a Kronecker product cannot be in Cartesian product and vice versa. i.e., these two products have no common edges. This leads to the fact that the two products have different structures. But in the case of paths, both products are bipartite. The graph $\mathrm{P}_{\mathrm{m}} \times \mathrm{P}_{\mathrm{n}}$ is also known as an $\mathrm{m} \times \mathrm{n}$ grid graph.

In this paper, we compare the cp-number and dc-number of $P_{m} \wedge P_{n}$ and $P_{m} \times P_{n}$ for $m=2,3$ and 4. The graphs $P_{4} \times P_{n}$ and $\mathrm{P}_{4} \wedge \mathrm{P}_{\mathrm{n}}$ are shown in figure 2(a) and 2(b) respectively.

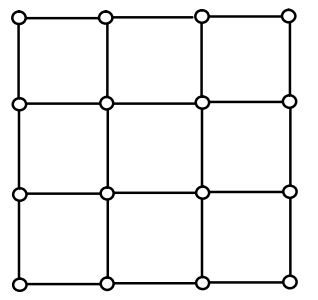

(a)

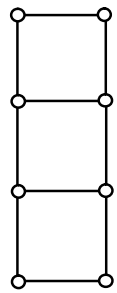

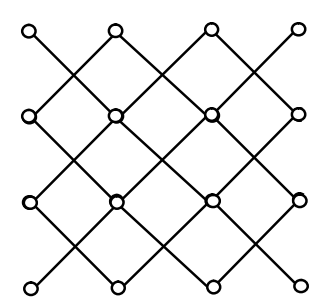

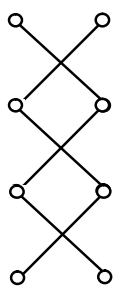

(b)

Figure 2

\section{Prior Results}

Conjecture 2.1 (Vizing's conjecture): For any two graphs $\mathrm{G}$ and $\mathrm{H}$, $\gamma(\mathrm{G} \times \mathrm{H}) \geq \gamma(\mathrm{G}) \gamma(\mathrm{H})$

Observation 2.2 [1]: For any bipartite graph $G, \operatorname{cpn}(G)=2$.

Proposition 2.3 [10]: For $n \geq 3$, 
A Comparison on the Bounds ...

$$
\gamma\left(P_{n}\right)=\left\{\begin{array}{l}
\frac{n}{3}, \quad \text { if } n \equiv 0(\bmod 3) \\
\frac{(n+2)}{3}, \text { if } n \equiv 1(\bmod 3) \\
\frac{(n+1)}{3}, \text { if } n \equiv 2(\bmod 3)
\end{array}\right.
$$

Proposition 2.4 [1]: For $n \geq 3$,

$$
\gamma_{\mathrm{ch}}\left(P_{\mathrm{n}}\right)=\left\{\begin{array}{l}
\frac{(\mathrm{n}+3)}{3}, \text { if } \mathrm{n} \equiv 0(\bmod 3) \\
\frac{(\mathrm{n}+2)}{3}, \text { if } \mathrm{n} \equiv 1(\bmod 3) \\
\frac{(\mathrm{n}+4)}{3}, \text { if } \mathrm{n} \equiv 2(\bmod 3)
\end{array}\right.
$$

Theorem 2.5 [11]: If $\mathrm{G}_{1}$ and $\mathrm{G}_{2}$ are connected graphs having no odd cycle then $G_{1} \wedge G_{2}$ has exactly two connected components.

Theorem 2.6 [12]: For $\mathrm{n} \geq 2$,

$$
\gamma\left(P_{2} \times P_{n}\right)=\left\{\begin{array}{l}
\frac{(n+1)}{2}, \text { if } n \text { is odd } \\
\frac{(n+2)}{2}, \text { if } n \text { is even }
\end{array}\right.
$$

\section{CP-Number of Kronecker Product and Cartesian Product}

Proposition 3.1: For $\mathrm{n}, \mathrm{m} \geq 2$,

(i) $\operatorname{cpn}\left(\mathrm{P}_{\mathrm{m}} \wedge \mathrm{P}_{\mathrm{n}}\right)=2$

(ii) $\operatorname{cpn}\left(\mathrm{P}_{\mathrm{m}} \times \mathrm{P}_{\mathrm{n}}\right)=2$

Proof: The proof follows from the fact that both $P_{m} \wedge P_{n}$ and $P_{m} \times P_{n}$ are bipartite.

\section{Dom-Chromatic Number in Kronecker Product}

Proposition 4.1: For $\mathrm{n} \geq 3$, 
T N Janakiraman and M Poobalaranjani

$$
\gamma_{c h}\left(P_{2} \wedge P_{n}\right)= \begin{cases}\gamma\left(P_{2} \times P_{n}\right)+1=\frac{2 n}{3}+1, & \text { if } n \equiv 0(\bmod 3) \\ \gamma\left(P_{2} \times P_{n}\right)=\frac{2(n-1)}{3}+2, & \text { if } n \equiv 1(\bmod 3) \\ \gamma\left(P_{2} \times P_{n}\right)+1=\frac{2(n-2)}{3}+3, & \text { if } n \equiv 2(\bmod 3)\end{cases}
$$

Proof: It is evident from figure 3 that $P_{2} \wedge P_{n}=2 P_{n}$ and hence the result follows.

$$
P_{2} \wedge P_{n}:
$$

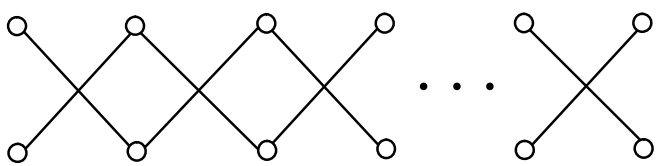

Figure 3

Proposition 4.2: For $n \geq 3, \gamma_{c h}\left(P_{3} \wedge P_{n}\right)=\gamma\left(P_{3} \wedge P_{n}\right)=n$

Proof: We show the result for $\mathrm{n}=3,4,5,6,7$ and generalize the result for $n \geq 8$.

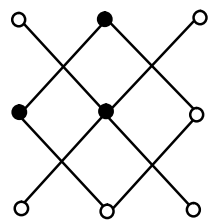

(a)

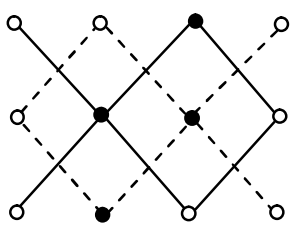

(b)

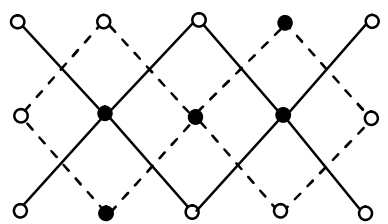

(c)

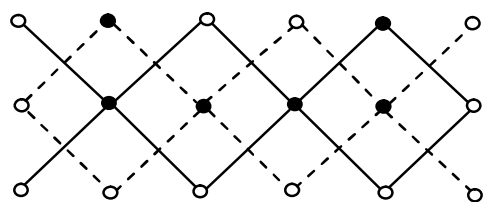

(d)

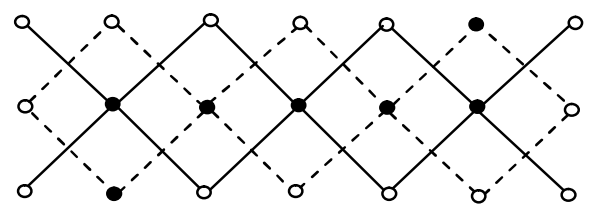

(e)

Figure 4 
If $\mathrm{n}=3$, then $\mathrm{P}_{3} \wedge \mathrm{P}_{3}$ is $\mathrm{K}_{1,4} \cup \mathrm{C}_{4}$ as shown in figure 4(a). Thus, it can be seen that $\gamma_{\mathrm{ch}}\left(\mathrm{P}_{3} \wedge \mathrm{P}_{3}\right)=\gamma\left(\mathrm{P}_{3} \wedge \mathrm{P}_{3}\right)=3$.

If $\mathrm{n}=4$, then the two components of $\mathrm{P}_{3} \wedge \mathrm{P}_{4}$ are shown in figure 4(b). The copies are identical and disjoint. For each copy, the domination number as well as the dc-number is 2 and hence, $\gamma_{\mathrm{ch}}\left(\mathrm{P}_{3} \wedge \mathrm{P}_{4}\right)=4$.

If $n=5$, then the two components of $P_{3} \wedge P_{5}$ are shown in figure $4(\mathrm{c})$. For one component the domination number is 2 and for the other component, the domination number and the dc-number are the same as 3 . Hence $\gamma\left(P_{3} \wedge P_{5}\right)=\gamma_{c h}\left(P_{3} \wedge P_{5}\right)=5$.

To see the pattern we take $\mathrm{n}=6$ and 7 .

Let $\mathrm{n}=6$. The graph $\mathrm{P}_{3} \wedge \mathrm{P}_{6}$ contains two identical components as shown in figure $3(\mathrm{~d})$. For each component, the domination number and the dc-number is 3 and hence, $\gamma_{c h}\left(P_{3} \wedge P_{4}\right)=\gamma_{c h}\left(P_{3} \wedge P_{6}\right)=6$.

Let $\mathrm{n}=7$. The graph $\mathrm{P}_{3} \wedge \mathrm{P}_{7}$ contains two components as shown in figure $4(\mathrm{~d})$. For one component, the domination number is 3 and for the other component, the domination number and the dcnumber are the same as 4 . Hence, $\gamma\left(P_{3} \wedge P_{7}\right)=\gamma_{c h}\left(P_{3} \wedge P_{7}\right)=7$. Hence $\gamma_{c h}\left(P_{3} \wedge P_{7}\right)=\gamma\left(P_{3} \wedge P_{7}\right)=7$.

In general, when $n$ is even, $P_{3} \wedge P_{n}$ has two identical components as given in figure 5 .

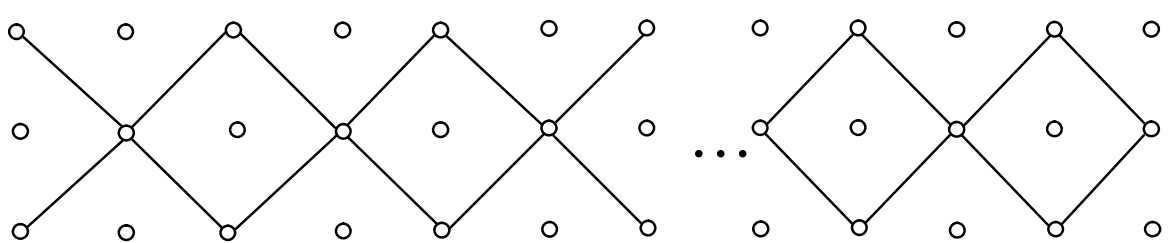

Figure 5

The domination number of the components can be obtained by taking the vertices in the middle row. (Refer figure 6). The number of vertices in the middle row equals $\left(\frac{\mathrm{n}}{2}\right)$. 


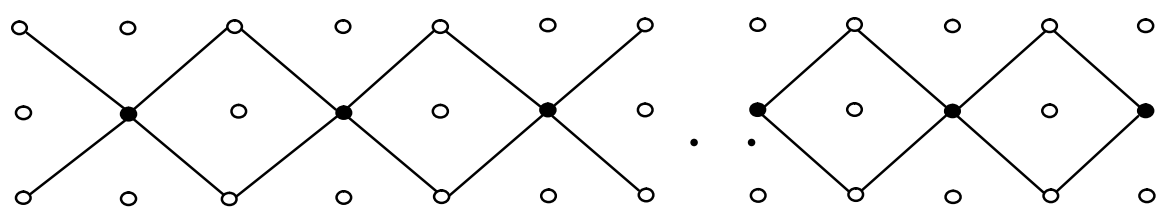

Figure 6

Now in one component, change the dominating vertex in the last column to the column before the last (Refer figure 7).

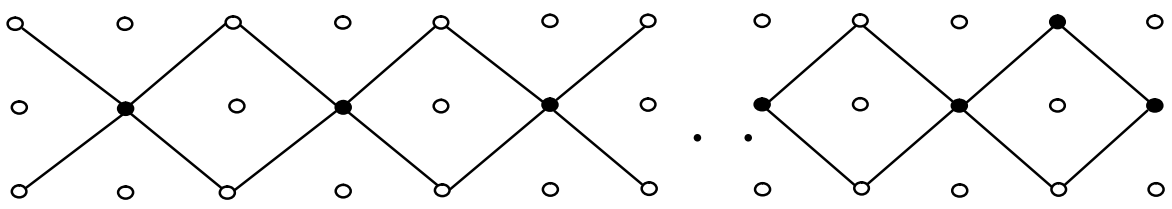

Figure 7

This rearrangement of the dominating vertex yields a dc-set of the component and of cardinality equals the domination number of the component. Taking the union of this set together with a minimum dominating set of the other component gives a minimum dc-set. Thus, $\gamma\left(P_{3} \wedge P_{n}\right)=\gamma\left(P_{3} \wedge P_{n}\right)=2\left(\frac{n}{2}\right)=n$.

Suppose that $\mathrm{n}$ is odd. The two components are given in figure 8 and 9.

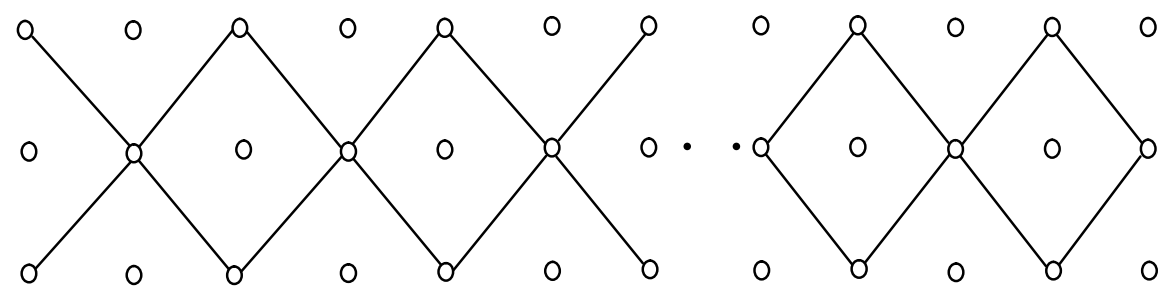

Figure 8 


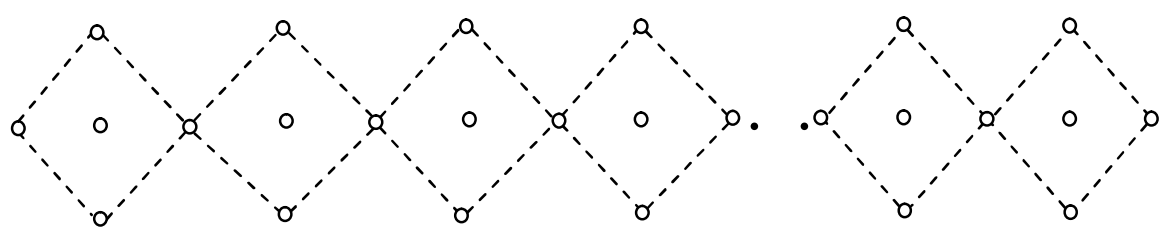

Figure 9

As before, the vertices in the middle row will form a $\mathrm{y}$-set. The domination number of the component in figure 8 is $\left(\frac{\mathrm{n}-1}{2}\right)$ and for the component in figure 8 is $\left(\frac{\mathrm{n}+1}{2}\right)$. In the $\mathrm{\gamma}$-set, replace vertex in the last column of figure 9 by a vertex adjacent to it, then the $\gamma$-set becomes a $\gamma_{c h}$-set.

Thus, $\gamma_{\mathrm{ch}}\left(\mathrm{P}_{3} \wedge \mathrm{P}_{\mathrm{n}}\right)=\mathrm{n}$.

Proposition 4.3: For $\mathrm{n} \geq 4$,

$$
\gamma_{c h}\left(P_{4} \wedge P_{n}\right)= \begin{cases}\gamma\left(P_{4} \wedge P_{n}\right)=n, & \text { if } n \equiv 0(\bmod 4) \\ \gamma\left(P_{4} \wedge P_{n}\right)=n+1, & \text { if } n \equiv 1,3(\bmod 4) \\ \gamma\left(P_{4} \wedge P_{n}\right)=n+2, & \text { if } n \equiv 2(\bmod 4)\end{cases}
$$

Proof: The graph $\mathrm{P}_{4} \wedge \mathrm{P}_{4}$ is given in figure 10(a). It has two identical components and one of its components is given in figure 10(b). The domination number as well as the dc-number of the component is 2 . 


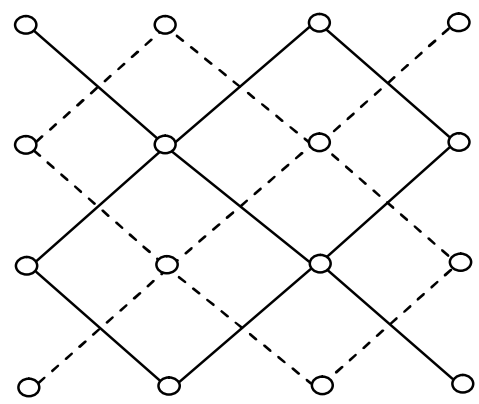

(a)

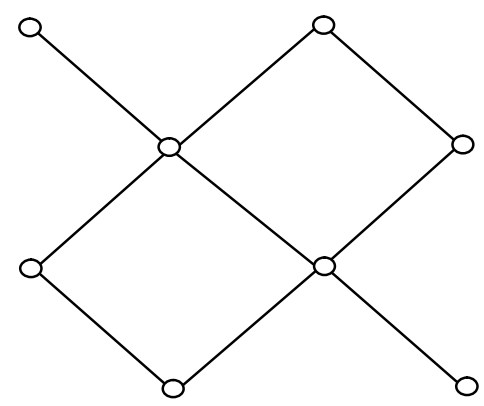

(b)

Figure 10

In figure 11(a), (b) and (c), one of the components of $P_{4} \wedge P_{5}, P_{4} \wedge P_{6}$, $\mathrm{P}_{4} \wedge \mathrm{P}_{7}$ are shown. We partition the graphs into $4 \times 4$ blocks. The graph $\mathrm{P}_{4} \wedge \mathrm{P}_{5}$ it is partitioned into a $4 \times 4$ and a $4 \times 1$ block, the graph $\mathrm{P}_{4} \wedge \mathrm{P}_{6}$ is partitioned into $4 \times 4$ and a $4 \times 2$ block and the graph $\mathrm{P}_{4} \wedge \mathrm{P}_{7}$ is partitioned into $4 \times 4$ and a $4 \times 3$ block.

Consider $\mathrm{P}_{4} \wedge \mathrm{P}_{5}$. The first block is dominated by 2 adjacent vertices. The second block has two isolated vertices and it can be dominated by a vertex in the first block.

Thus, $\gamma_{\text {ch }}\left(P_{4} \wedge P_{5}\right)=2 \times 3=6$.

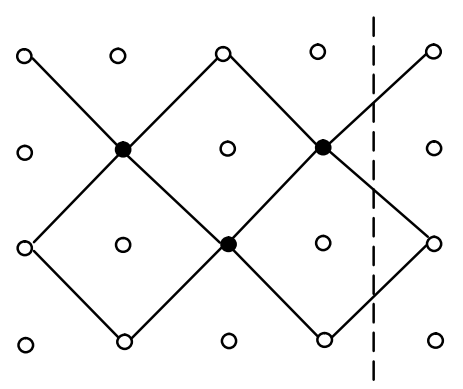

(a)

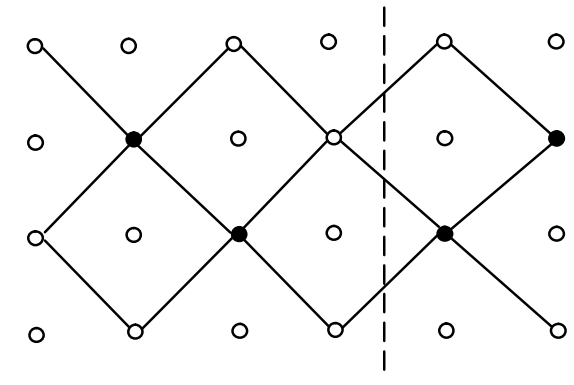

(b 


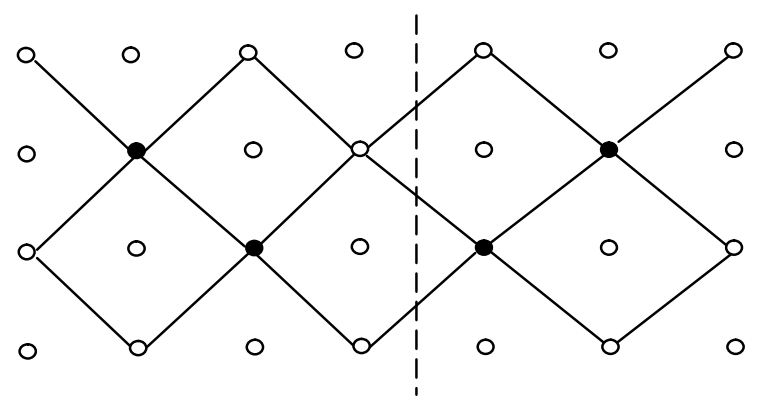

(c)

Figure 11

Consider $\mathrm{P}_{4} \wedge \mathrm{P}_{6}$. The first block is dominated by 2 adjacent vertices. The second block has an induced path $\mathrm{P}_{4}$ and is dominated by 2 vertices in the second block. Thus $\gamma_{\mathrm{ch}}\left(\mathrm{P}_{4} \wedge \mathrm{P}_{6}\right)=2 \times 4=8$.

Consider $\mathrm{P}_{4} \wedge \mathrm{P}_{7}$. The first block is dominated by 2 adjacent vertices and the second block is also dominated by 2 adjacent vertices. Thus $\gamma_{\text {ch }}\left(\mathrm{P}_{4} \wedge \mathrm{P}_{7}\right)=2 \times 4=8$.

Let $B_{1}, B_{2}$ and $B_{3}$ represent the blocks with 1 column, 2 columns and 3 columns respectively.

In general, when a component is partitioned in to $4 \times 4$ blocks, if $\mathrm{n} \equiv 0(\bmod 4)$, then using the fact that each block is dominated by 2 adjacent vertices, dc-number of the component $=$ domination number $=2\left(\frac{\mathrm{n}}{4}\right)$.

Therefore, $\gamma_{c h}\left(P_{4} \wedge P_{n}\right)=2\left(2\left(\frac{n}{4}\right)\right)=n$.

Suppose that $\mathrm{n} \equiv 1(\bmod 4)$. Then the number of $4 \times 4$ blocks in each component $=\left(\frac{\mathrm{n}-1}{4}\right)$. Each component has one $\mathrm{B}_{1}$ block. 
Hence, dc-number of each component $=$ domination number $=$ $2\left(\frac{\mathrm{n}-1}{4}\right)+1$.

Thus, $\gamma_{\text {ch }}\left(P_{4} \wedge P_{n}\right)=2\left(2\left(\frac{n-1}{4}\right)+1\right)=n+1$.

When $\mathrm{n} \equiv 2(\bmod 4)$, the component has a $\mathrm{B}_{2}$ block and when $\mathrm{n} \equiv 3(\bmod 4)$, the component has a $\mathrm{B}_{3}$ block, and in both cases the blocks are dominated by two vertices. Hence, if $\mathrm{n} \equiv 2(\bmod 4)$, then dc-number of each component $=$ domination number $=$ $2\left(\frac{\mathrm{n}-2}{4}\right)+2$.

Thus, $\gamma_{\text {ch }}\left(P_{4} \wedge P_{n}\right)=2\left(2\left(\frac{n-2}{4}\right)+2\right)=n+2$.

If $\mathrm{n} \equiv 3(\bmod 4)$, then

dc-number of each component $=$ domination number $=$ $2\left(\frac{\mathrm{n}-3}{4}\right)+2$.

Thus, $\gamma_{c h}\left(P_{4} \wedge P_{n}\right)=2\left(2\left(\frac{n-3}{4}\right)+2\right)=n+1$.

\section{Dom-Chromatic Number in Cartesian Product}

For Cartesian product, the dominating set structure is based on the result given in [12].

Proposition 5.1: For $\mathrm{n} \geq 2$,

$$
\gamma_{c h}\left(P_{2} \times P_{n}\right)= \begin{cases}\gamma\left(P_{2} \times P_{n}\right)=\frac{n+2}{2}, & \text { if } n \text { is even } \\ \gamma\left(P_{2} \times P_{n}\right)+1=\frac{n+3}{2}, & \text { if } n \text { is odd }\end{cases}
$$

Proof: The minimum dominating set is formed by choosing the vertices diagonally in the alternate columns and beginning from the first column. If $\mathrm{n}$ is odd, then we have one of the following minimum domination structures as given in figure 12 (a), when $\mathrm{n} \equiv 3(\bmod 4)$ and figure $12(\mathrm{~b})$, when $\mathrm{n} \equiv 1(\bmod 4)$. 


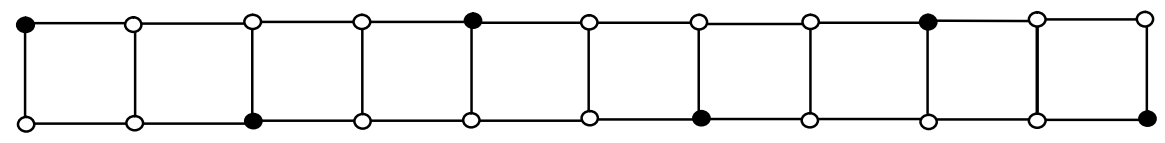

(a) $(\mathrm{n}=11 \equiv 3(\bmod 4))$

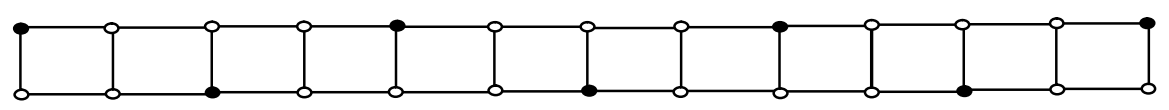

(b) $(\mathrm{n}=13 \equiv 1(\bmod 4))$

Figure 12

A dc-set can be obtained by choosing any vertex from the remaining vertices. In other words,

$$
\text { dc-number }=\text { domination number }+1 \text {. }
$$

Thus, $\gamma_{c h}\left(P_{2} \times P_{n}\right)=\gamma\left(P_{2} \times P_{n}\right)+1$

$$
=\left(\frac{\mathrm{n}+1}{2}\right)+1=\left(\frac{\mathrm{n}+3}{2}\right)
$$

If $\mathrm{n}$ is even, then we have one of the following minimum domination structures given in figure $13(\mathrm{a})$, when $\mathrm{n} \equiv 0(\bmod 4)$ and figure $13(\mathrm{~b})$, when $\mathrm{n} \equiv 2(\bmod 4)$.

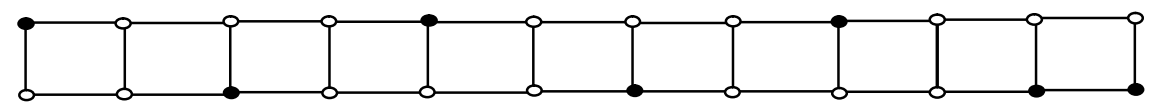

(a) $(\mathrm{n}=12 \equiv 0(\bmod 4))$

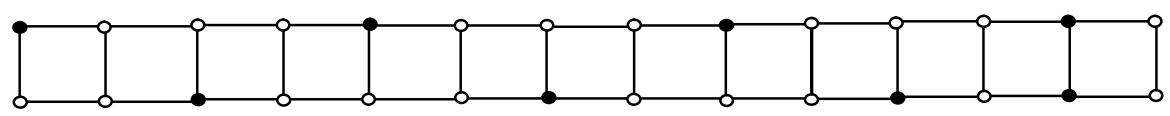

(b) $(\mathrm{n}=14 \equiv 2(\bmod 4))$

Figure 13

Clearly the $\gamma$-set shov 13 is a dc-set. Hence, $\gamma_{c h}\left(P_{2} \times P_{n}\right)=\gamma\left(P_{2} \times P_{n}\right)=\left(\frac{n+2}{2}\right)$.

Proposition 5.2: For $\mathrm{n} \geq 3$, 


$$
\gamma_{c h}\left(P_{3} \times P_{n}\right)=\left\{\begin{array}{l}
\gamma\left(P_{3} \times P_{n}\right)+1=\left\lfloor\frac{(3 n+4)}{4}\right\rfloor+1, \text { if } n \text { is odd } \\
\gamma\left(P_{3} \times P_{n}\right)=\left\lfloor\frac{(3 n+4)}{4}\right\rfloor, \quad \text { if } n \text { is even }
\end{array}\right.
$$

Proof: When $\mathrm{n}$ is odd, the domination structure is shown in figure $14(\mathrm{a})$, when $\mathrm{n} \equiv 3(\bmod 4)$ figure $14(\mathrm{~b})$, when $\mathrm{n} \equiv 1(\bmod 4)$.

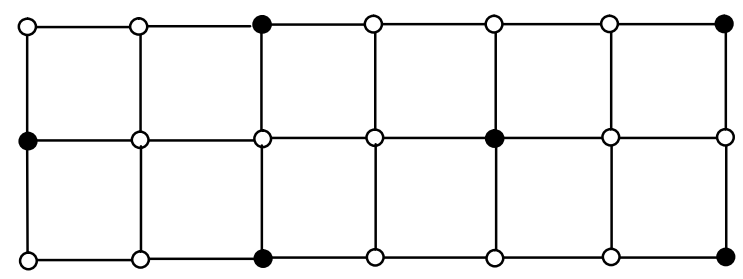

(a) $(\mathrm{n}=7 \equiv 3(\bmod 4))$

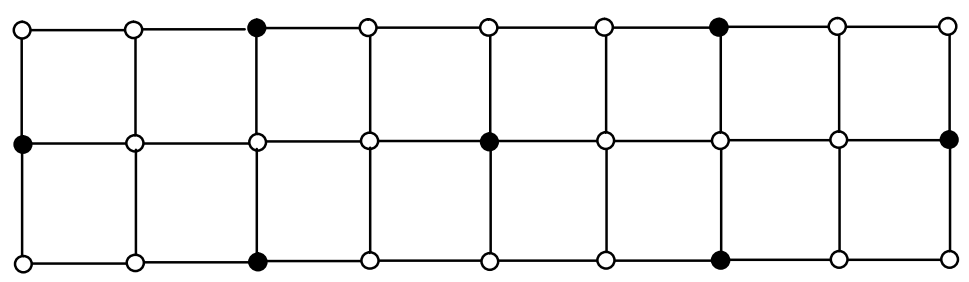

(b) $(\mathrm{n}=9 \equiv 1(\bmod 4))$

Figure 14

By adding any vertex to the given dominating set we get a dc-set.

Hence, if $n$ is odd, $\gamma_{c h}\left(P_{3} \times P_{n}\right)=\gamma\left(P_{3} \times P_{n}\right)+1=\left\lfloor\frac{3 n+4}{4}\right\rfloor+1$.

If $\mathrm{n}$ is even, the domination structure is shown in figure 15 (a), when $\mathrm{n} \equiv 0(\bmod 4)$ and figure $15(\mathrm{~b})$, when $\mathrm{n} \equiv 2(\bmod 4)$.

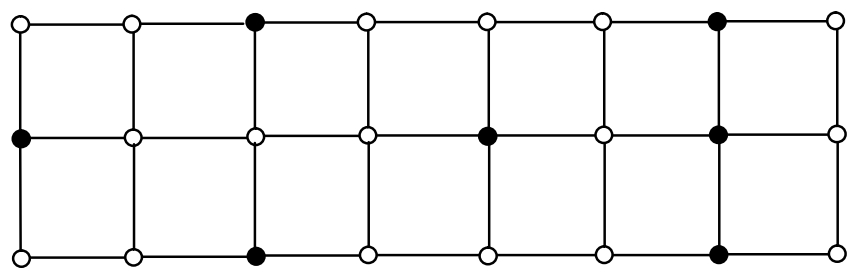

(a) $(\mathrm{n}=8 \equiv 0(\bmod 4))$ 


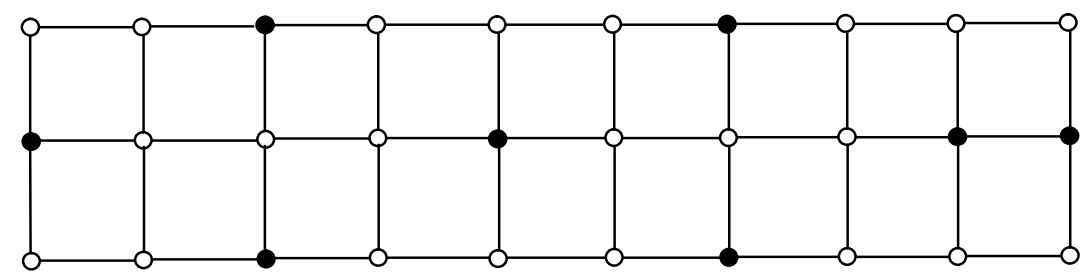

(b) $(\mathrm{n}=10 \equiv 2(\bmod 4))$

Figure 15

The minimum dominating set itself is a dc-set. Hence, if $\mathrm{n}$ is even,

$\gamma_{c h}\left(P_{3} \times P_{n}\right)=\gamma\left(P_{3} \times P_{n}\right)=\left\lfloor\frac{3 n+4}{4}\right\rfloor$.

Proposition 5.3: For $\mathrm{n} \geq 4$,

$$
\gamma_{c h}\left(P_{4} \times P_{n}\right)=\left\{\begin{array}{l}
\gamma\left(P_{4} \times P_{n}\right)=n+1, \text { if } n=5,6,9 \\
\gamma\left(P_{4} \times P_{n}\right)+1=n+1, \text { otherwise }
\end{array}\right.
$$

Proof: The proof is trivial. The domination structure is given in figure 16.

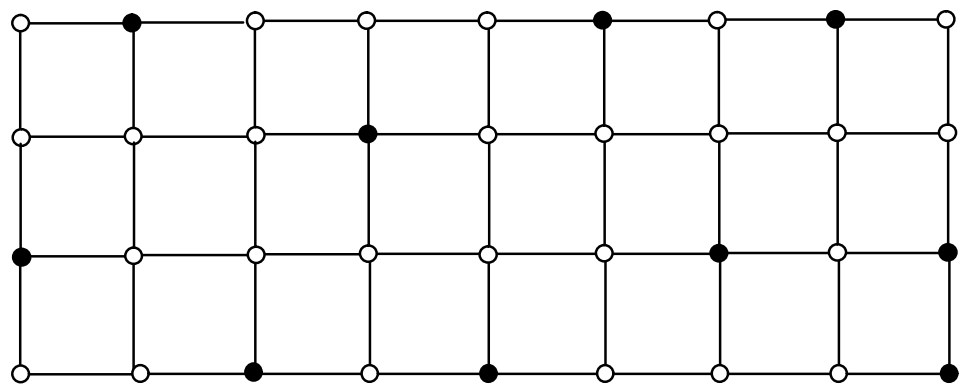

\section{Comparison of DC- $\Gamma$ Figure 16 Cartesian Product and Kronecker Product}

For any bipartite graph, the dc-number is either the domination number or greater than the domination number by one. Since both the products are bipartite graph, this is applicable to these products 
also. Though their cp-numbers are the same, the products cannot be totally classified as graphs whose domination number and the dc-number are the same or the dc-number is greater than the domination number by 1 .

\section{References}

[1] T N Janakiraman and M Poobalaranjani, Dom-Chromatic sets in bipartite graphs. Int. J. Engg. Sci. Advanced Computing and Bio-technology, vol. 1, pp. 80-95, 2010.

[2] T N Janakiraman and M Poobalaranjani, Dom-Chromatic sets of graphs. Int. J. Engg. Sci. Advanced Computing and Bio-technology, vol. 2, pp. 88-103, 2011.

[3] G A Dirac, A property of 4-chromatic graphs and some remarks on critical graphs. J. London Math. Soc., vol. 27, pp. 85-92, 1952.

[4] T N Janakiraman and M Poobalaranjani, On the chromatic preserving sets. Int. J. Engg. Sci. Advanced Computing and Bio-technology, vol. 1, pp. 29- 42, 2010.

[5] W Imrich and H Izbicki, Associative products of graphs, Monatsh. Math., vol. 80, pp. 277-281, 1975.

[6] P M Weishsel, The Kronecker product of graphs. Proc. Amer. Math. Soc., vol. 13, pp. 470-52, 1962.

[7] $\mathrm{S} \mathrm{H}$ Shapiro, The embedding of graphs in cubes and design of sequential relay circuits. Bell. Telephone Lab. Memorandum (unpublished report), 1953.

[8] G Sabidussi. Graph multiplication. Math. Z., vol. 72, pp. 446-457, 1960.

[9] H H Teh and H P Yap, Some construction problems of homogeneous graphs. Bull. Math. Soc., Nanyang Univ., pp. 164-196, 1964.

[10] T W Haynes, S T Hedetniemi and P J Slater, Fundamentals of Domination in Graphs, Marcel Dekker Inc., New York, 1998.

[11] T Sitthiwirattham, Independednt and vertex covering number of Kronecker product of $\mathrm{K}_{\mathrm{m}, \mathrm{n}}$, Applied Mathematical Sciences, vol. 6, pp. 14031408, 2012.

[12] T Y Chang, W E Clark and E O Hare, Domination numbers of complete grid graphs-I, Dec. 19, 1995. 